\title{
Estratégia omnichannel e a experiência do usuário: um estudo na rede social digital LinkedIn
}

\section{Omnichannel strategy and user experience: a study at Linkedln}

\author{
Bruno Soares Ramos ${ }^{1}$, Arthur Ferreira Campos ${ }^{2}$, Eliane Bezerra Paiva ${ }^{3}$, Gustavo Henrique de Araújo Freire ${ }^{4}$, \\ Marckson Roberto Ferreira de Sousa ${ }^{5}$ \\ ${ }^{1}$ Universidade Federal da Paraíba (UFPB), João Pessoa, Paraíba, Brasil. ORCID: https://orcid.org/0000-0002-3336-5407 \\ 2 Universidade Federal da Paraíba (UFPB), João Pessoa, Paraíba, Brasil. ORCID: https://orcid.org/0000-0001-6561-1951 \\ ${ }^{3}$ Universidade Federal da Paraíba (UFPB), João Pessoa, Paraíba, Brasil. ORCID: https://orcid.org/0000-0002-9925-0143 \\ 4 Universidade Federal do Rio de Janeiro (UFRJ), Rio de Janeiro, Rio de Janeiro, Brasil. ORCID: https://orcid.org/0000-0002-5540-4630 \\ ${ }^{5}$ Universidade Federal da Paraíba (UFPB), João Pessoa, Paraíba, Brasil. ORCID: https://orcid.org/0000-0003-2001-1631
}

Autor para correspondência/Mail to: Arthur Ferreira Campos, arthurfcampos94@gmail.com

Recebido/Submitted: 17 de maio de 2021; Aceito/Approved: 27 de junho de 2021

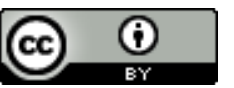

Copyright (c) 2021 Ramos, Campos, Paiva, Freire \& Sousa. Todo o conteúdo da Revista (incluindo-se instruções, política editorial e modelos) está sob uma licença Creative Commons Atribuição 4.0 Internacional. Ao serem publicados por esta Revista, os artigos são de livre uso em ambientes educacionais, de pesquisa e não comerciais, com atribuição de autoria obrigatória. Mais informações em http://revistas.ufpr.br/atoz/about/submissions\#tcopyrightNotice.

\begin{abstract}
Resumo
Introdução: a informação está presente nos diversos meios e recursos tecnológicos existentes, sendo utilizada tanto para uma compra online como para redigir um texto numa rede social digital, expondo opinião sobre um determinado fato ou assunto. Utiliza a estratégia omninichannel para entender a gestão integrada de canais de comunicação no uso simultâneo e na interligação desses diferentes canais, analisando a experiência do usuário e sua satisfação em relação à rede social digital Linkedln. Objetiva compreender a importância da estratégia omninichannel para a experiência do usuário na rede social digital Linkedln. Método: o percurso metodológico é alicerçado pela abordagem qualitativa e utiliza formulário online, averiguando a satisfação no Linkedln quanto à estratégia omninichannel e à experiência do usuário. Resultados: os resultados apontam a consolidação de um público especializado e experiente na utilização da rede social digital Linkedln, constatando possibilidades de melhorias, expondo as dificuldades de uso enfrentadas e conhecendo a estratégia omninichannel como forma de integração entre o usuário e os recursos inerentes à estratégia. Conclusões: a partir do alcance do objetivo do artigo, considera que o relacionamento estratégico possibilitado pela omninichannel é desenvolvido mediante a integração de conteúdo disseminado nos canais de comunicação juntamente com a integração entre os próprios usuários da informação.
\end{abstract}

Palavras-chave: Estratégia omnichannel; Experiência do Usuário; Redes sociais digitais; LinkedIn.

\begin{abstract}
Introduction: the information is present in the various existing technological means and resources, being used both for online purchase and for writing a text on a digital social network, exposing an opinion on a certain fact or subject. It uses the omnichannel strategy to understand the integrated management of communication channels in the simultaneous use and interconnection of these different channels, analyzing the user experience and their satisfaction concerning LinkedIn social media. It aims to understand the importance of the omnichannel strategy for the user experience in Linkedln social media. Method: the methodological path is based on the qualitative approach and uses an online form to ascertain satisfaction on Linkedln regarding the omnichannel strategy and the user experience. Results: the results point to the consolidation of a specialized and experienced public in the use of the Linkedln social media, realizing possibilities for improvements exposing the difficulties of use faced and knowing the omnichannel strategy as a way of integration between the user and the resources inherent to the strategy. Conclusions: it considers that the strategic relationship made possible by omnichannel is developed through the integration of content disseminated in the communication channels given the integration between the information users themselves.
\end{abstract}

Keywords: Omnichannel Strategy; User Experience; Social Media; Linkedln.

\section{INTRODUÇÃO}

O contato do ser humano com ambientes informacionais é uma prática proeminente na sociedade pós-moderna. O discurso de que a informação está em todo lugar se torna constante quando o sujeito se depara com recursos e dispositivos de informação no seu dia a dia. Segundo Araújo (2018), pesquisar, produzir e consumir conteúdo, assistir a um documentário, postar um texto ou uma imagem em ambiente web são práticas comuns ao sujeito atualmente.

Uma das características da sociedade pós-moderna é a necessidade de se conectar, interagir e compartilhar informações nas redes sociais digitais, estabelecendo relações entre pessoas, conectando-as, formando comunidades ou redes de relacionamentos, com uma comunicação rápida e interativa. Tomaél e Marteleto (2006) consideram que uma rede social digital é o conjunto de pessoas conectadas por relações sociais ou profissionais que permitem o compartilhamento de informações, e assim constituem uma ligação social. 
Segundo Van Dijck (2016), o crescimento da Internet, aliado ao acesso das mídias sociais, possibilita aos indivíduos o fortalecimento das relações sociais digitais, proporcionando solidez no desenvolvimento das tecnologias de comunicação que agora fazem parte das práticas sociais cotidianas, marcadas pelo acesso rápido às informações que estão sendo compartilhadas entre as pessoas. Desse modo, a comunicação "face to face" ${ }^{1}$ está sendo complementada pela utilização das redes sociais digitais, potencializando, na era digital, a migração do "social" para o "virtual" e para o "digital". Com isso, ferramentas de interação estão sendo criadas para dinamizar a comunicação humana e a estratégia omnichannel é uma dessas. Essa estratégia é utilizada em experiências de compra, ampliando e buscando integrar vários canais para o cliente, entregando uma gestão integrada em multicanais (Guissoni, 2017). Segundo Fonseca (2017), omnichannel busca disponibilizar informações em diferentes canais de comunicação, possibilitando a relação entre on-line e off-line, contribuindo na experiência do cliente. A experiência do usuário, nessa integração, é um quesito de satisfação necessário para análise, haja vista que uma organização deve também preocupar-se com a satisfação do sujeito ao entregar um determinado serviço.

A rede social digital LinkedIn utiliza a estratégia omnichannel ao utilizar e interligar simultaneamente diferentes canais de comunicação, sendo um ambiente voltado aos negócios e à procura de profissionais qualificados para o mercado de trabalho. Essa estratégia é relevante e apresenta uso potencial no escopo da Ciência da Informação, especialmente nos estudos de usuários. Neste contexto, apresenta-se a seguinte questão: como ocorre a aceitação da estratégia omnichannel aliada à usabilidade e experiência do usuário na rede social digital LinkedIn? Frente a isso, o objetivo do estudo é compreender a importância da estratégia omnichannel para a experiência do usuário na rede social digital LinkedIn. O percurso metodológico deste estudo caminha na percepção da relação da estratégia omnichannel e da experiência do usuário, tendo a rede social digital LinkedIn como campo de estudo; obtivemos 136 respostas de usuários do LinkedIn sobre satisfação, usabilidade e percepção omnichannel, mediante a plataforma Google Forms.

A justificativa do estudo é pautada na ausência de publicações sobre a estratégia omnichannel na Ciência da Informação e, sendo isso uma estratégia de gestão integrada, subsidia a construção de conhecimento sobre gestão no campo da Ciência da Informação. Dessa forma, a rede social digital LinkedIn demonstra potencial considerável para estudos de satisfação com a experiência do usuário, usabilidade, gestão e sobre esse uso simultâneo e interligado de diferentes canais de comunicação coerente com a estratégia omnichannel. Esta pesquisa possui financiamento da Coordenação de Aperfeiçoamento de Pessoal de Nível Superior (CAPES) Código de financiamento 001.

\section{A relação empresa-consumidor nas redes sociais digitais}

Com a facilidade provocada pela web e pelas plataformas digitais, as redes sociais digitais auxiliam de forma significativa no marketing e exibição das empresas, proporcionando uma oportunidade para as organizações que estão interessadas na pesquisa com os consumidores e usuários, tornando-se uma grande aliada para conhecer o seu público. Segundo Torres (2009), quando a empresa participa das redes sociais digitais, torna-se muito mais próxima de seu consumidor e o próprio consumidor acaba "orientando" a empresa, informando suas necessidades e expondo as tendências que o mercado exige. A utilização social da tecnologia possibilitou o surgimento da cibercultura e, com isso, a possibilidade de formação de novas relações entre comunidades virtuais e usuários de Internet, favorecendo o estabelecimento de interações entre os assuntos ou atividades de interesse comum; sendo assim, esses fluxos de informação são recorrentes e podem superar as interações presenciais (Lemos, 2010). As redes sociais digitais são ferramentas de Marketing de Relacionamento, que provocam grandes mudanças de interação nas relações empresa-consumidor. Esse relacionamento deve ser de forma dinâmica, transmitindo segurança nas informações prestadas. Destaca-se que a empresa seja responsável, autêntica e sincera em sua comunicação nas redes sociais digitais, respondendo aos consumidores e adotando ações transparentes de correção para quando algo sair de forma inesperada (Torres, 2009).

De acordo Costa e Picchi (2017), as empresas começaram a utilizar as redes sociais digitais para ações estratégicas, tornando-as canais de divulgação e comercialização de marcas, produtos, serviços e propagação de publicidades. A ausência de uso das redes sociais digitais pelas empresas, as quais possibilitam manter um diálogo com maior praticidade, pode gerar insatisfação nos seus clientes. Ainda segundo Costa e Picchi (2017), há a possibilidade de frustração do usuário ao não encontrar a página de determinada empresa em uma mídia social, haja vista que a possibilidade de interação e de compartilhamento se limita bastante. Com o aumento do número de usuários nas redes sociais digitais, muitas empresas começaram a adotar a estratégia omnichannel como gestão integrada. Podendo obter vantagem competitiva perante empresas concorrentes, a omnichannel possibilita maior agilidade no relacionamento com o cliente, permitindo rapidez na tomada de decisão de seus usuários e/ou consumidores, facilitando a entrega de um produto ou recurso aos seus usuários.

\section{Estratégia channel}

A evolução tecnológica possibilita a crescente utilização dos dispositivos móveis, como smartphones, notebooks e tablets por sujeitos informacionais. A necessidade de se conectar ocasiona transformações na busca por informação,

\footnotetext{
${ }^{1}$ Tradução nossa: cara a cara; face a face; frente e frente; comunicação interpessoal.
} 
compra de serviços ou produtos e na navegação em ambientes informacionais. O consumidor omnichannel não busca apenas realizar compras em diversos canais integrados, trata-se de um usuário hiperconectado que procura desenvolver um relacionamento com as marcas em diversas plataformas, tendo uma experiência autêntica (Kotler, Kartajaya, \& Setiwan, 2017). Sendo assim, (Verhoef, Kannan, \& Inman, 2015, p. 176) afirmam que omnichannel está relacionada com a gestão sinérgica "dos variados canais de venda disponíveis e pontos de contato com o cliente, de forma que a experiência dos clientes através dos canais e o desempenho global destes sejam otimizados". Assim, a estratégia omnichannel possui como centro de suas ações o consumidor da informação, ou seja, o próprio usuário. Porém, o processo de modernização dos produtos e serviços potencializa o desejo por experiências marcantes, até mesmo memoráveis. Guissoni (2017) destaca que a omnichannel é uma estratégia de gestão integrada, analisando dados do consumidor de maneira sincronizada e multicanal, gerando uma vantagem competitiva, ou seja, o uso de diferentes dispositivos para execução de uma ação (compra, postagem, interação, entre outros) dinamiza o produto que as organizações devem entregar ao usuário, sincronizando cada vez mais os ambientes on-line e off-line. Fonseca (2017) o afirma que

Omnichannel é uma estratégia de uso simultâneo e interligado de diferentes canais de comunicação, com o objetivo de estreitar a relação entre on-line e off-line, aprimorando, assim, a experiência do cliente. Essa tendência do varejo permite a convergência do virtual e do físico.

É necessário ter uma estratégia bem alinhada para que o sucesso seja o catalisador de experiências customizadas, reconhecendo no usuário de informação o ponto de partida para o conhecimento do seu comportamento, do mapeamento dos dados disponíveis e, a partir deles, a efetivação da estratégia junto às ações que proporcionarão a satisfação do consumidor. Portanto, o gerenciamento das ações do cliente se torna uma das principais fontes de vantagem competitiva.

\section{Usabilidade e experiência do usuário}

Borko (1968) indica que a Ciência da Informação está relacionada com a usabilidade e acessibilidade, investigando as propriedades, comportamentos, fluxos e meios para o processamento da informação (Campos \& Oliveira, 2020). O autor, considerado clássico da área, já no final dos anos 1960 visualiza a interligação dessa ciência com a otimização de acesso e uso da informação para os sujeitos, o que contribui para a satisfação na experiência do usuário. As Tecnologias de Informação e Comunicação impulsionam transformações na interação dos sujeitos com o acesso à informação e a construção de conhecimento. A web, sendo um espaço de interação social, possibilita a produção e o consumo de conteúdo e, sendo assim, a necessidade de se estudar a satisfação do sujeito perante ferramentas ou estratégias digitais é bem-vinda. A estratégia omnichannel, utilizada por empresas como Magazine Luiza, Natura, Centauro, O Boticário e a Riachuelo ${ }^{2}$, contribui para o acesso a informações de maneira rápida, satisfatória e acessível.

Mediante a essas possibilidades, os recursos da web são os facilitadores para a gestão integrada da estratégia omnichannel. Dessa forma, esses recursos necessitam de análises de navegação, usabilidade e satisfação (experiência do usuário) e, neste artigo, realizamos um estudo com foco na satisfação em contexto a experiência do usuário e na usabilidade disposta nos recursos omnichannel (integração entre diversos canais de maneira interativa) da rede social digital LinkedIn.

A usabilidade envolve questões referentes ao grau com que o sistema satisfaz as necessidades e os requisitos dos sujeitos que o utilizam e o acessam (Nielsen, 1993). Pode-se dizer que a usabilidade define a facilidade com que os sujeitos utilizam um recurso com o objetivo de realizar uma tarefa específica, sendo investigada por métodos de mensuração e por estudos dos princípios recorrentes da eficiência de um recurso. Nielsen (1993) observa cinco atributos que são ligados à usabilidade. São eles: 1. facilidade de aprendizagem (Learnability) - o sistema deve ser fácil de aprender para que se possa começar a interagir rapidamente; 2. eficiência de uso (Efficiency) assim que se saiba como utilizar o sistema, deve haver condições para alcançar a maior produtividade possível; 3 . facilidade de memorização (Memorability) - deve haver simplicidade na retenção de como utilizar o sistema, para que após certo tempo, não se necessite aprender tudo novamente; 4. baixa taxa de erros (Errors) - deve haver uma baixa taxa de erros, e quando eles acontecerem, deve existir a capacidade de recuperação e; 5 . satisfação subjetiva (Satisfaction) - o sistema deve ser agradável de usar (Sousa, 2012). Para isso,

os usuários quando utilizam a web normalmente procuram informações que atendam aos seus interesses, mas preferem os websites que sejam fáceis de usar, possibilitando sua utilização sem necessidade de aprendizagem. Eles normalmente sentem-se satisfeitos quando alcançam seus objetivos de forma rápida e sem erros, esperando do sistema o máximo em termos de recuperação da informação. Com a crescente complexidade computacional dos métodos de interação, o foco passou a enfatizar a relação humano-computador e o desempenho do usuário (Sousa, 2012, p. 72).

O autor comenta que o desenvolvimento de ambientes informacionais digitais, adotando os princípios da usabilidade, é de grande importância para que se exibam conteúdos de forma satisfatória ao sujeito. Nesse contexto, estudar a usabilidade é conveniente com o estudo da experiência do usuário, visto que a satisfação

${ }^{2}$ Informação disponível no website da empresa Maplink: https://maplink.global/blog/empresas-utilizam-omnichannel-brasil/. 
do sujeito é uma necessidade na sociedade pós-moderna. E, considerando o diálogo realizado também com a omnichannel, verificamos uma possibilidade de convergência entre esses estudos. Utilidade, funcionalidade, usabilidade, design visual e competências individuais são uns dos procedimentos que subsidiam a experiência do usuário, porém não são os únicos. Ferreira (2018, p. 33) entende que a "[...] experiência do usuário e seus objetos de estudo ganham novos significados para a literatura; talvez por se tratar de um campo de pesquisa amplo e subjetivo, seu referencial teórico encontra-se em constante evolução." Essas discussões datam do século XIX e início do século XX, com a revolução industrial, quando se iniciaram os estudos sobre comportamento de usuários.

Buley (2013) indica que a experiência do usuário é influenciada por variadas áreas do conhecimento, tendo seu crescimento alicerçado ao avanço tecnológico e a concorrência dos produtos no mercado, necessitando o conhecimento das preferências e experiências das 'pessoas', com a finalidade de otimizar a produção de mercado. O autor ilustra que a experiência do usuário teve marcos históricos no Taylorismo, no Fordismo, nas ciências cognitivas, na computação pessoal, na web, nos dispositivos móveis, entre outros. Estabelecer um diálogo teórico que favoreça a interligação da omnichannel, da experiência do usuário e da usabilidade é uma tarefa importante para a construção de conhecimento na Ciência da Informação, mais especificamente no seu campo teórico contemporâneo da Gestão da Informação, conforme o discurso de Araújo (2018).

\section{PERCURSO METODOLÓGICO}

Minayo (2012) aponta a abordagem qualitativa ressaltando que a estruturação para os verbos de investigação 'compreender' e 'interpretar' e os substantivos 'experiência', 'vivência' e 'ação social' convergem com essa natureza de estudo. Diante disso, é elaborado uma abordagem para análise e levantamento de subsídios qualitativos. Realiza-se diálogo teórico entre a omnichannel, a experiência do usuário e a usabilidade, trazendo o discurso para a Ciência da Informação e destaca-se a necessidade de estudos subsequentes para solidificar esse diálogo. Neste artigo, apresenta-se uma pesquisa de satisfação com usuários da rede social digital LinkedIn conforme o embasamento teórico supracitado. Elaborou-se um questionário, via Google Forms, direcionado ao público brasileiro e dividido em três partes: a primeira contendo questões para o mapeamento do perfil do usuário com perguntas sobre gênero, faixa etária, escolaridade, ocupação e o setor de atuação do/a respondente; a segunda contendo perguntas sobre uso da rede social LinkedIn, satisfação e usabilidade para identificar a experiência do usuário; a terceira contendo perguntas sobre a aceitação ou não da omnichannel no LinkedIn. Antes do compartilhamento com o público geral usuário do LinkedIn, realizou-se um pré-teste com oito sujeitos usuários do LinkedIn. O pré-teste foi aplicado a pessoas vinculadas a Universidades Federais em âmbito graduação e pós-graduação (4) e pessoas vinculadas ao mercado de trabalho (4). A partir do pré-teste, constataram-se abordagens diretas e sucintas para as perguntas, como também um direcionamento melhor para um alcance de resultados positivos na fase de coleta de dados.

O questionário compartilhado com o público geral usuário do LinkedIn conteve 17 perguntas em Português-Brasil, sendo disponibilizado na web do dia $1^{\circ}$ do mês de dezembro de 2020 e encerrado no dia 22 do mês de janeiro de 2021. Cada respondente teve ciência do objetivo e do fundamento da pesquisa a partir da leitura do Termo de Consentimento Livre e Esclarecido antes de acessar a parte do questionário no Google Forms. No Termo, foram indicados os/as autores/as da pesquisa, o vínculo com a Universidade Federal da Paraíba (UFPB), assim como o vínculo dos autores/as com a disciplina de Usuários da Informação do Programa de Pós-Graduação em Ciência da Informação da UFPB. Também foi explicado no questionário, tanto na parte de experiência do usuário quanto na parte da estratégia omnichannel o conceito de cada estudo, a fim de informar ao respondente. Obteve-se um feedback de 136 respostas, visto que se optou por obter uma amostra intencional dentro do prazo que o questionário esteve aberto para respostas. Além da abordagem qualitativa, a pesquisa também assume a função descritiva, visto que os dados coletados são descritos discutindo com os resultados para a construção das considerações finais. A natureza da pesquisa é aplicada ao passo que se aplicou o questionário supracitado para a comunidade de usuários do LinkedIn por meio do compartilhamento desse questionário nos perfis pessoais dos/as autores/as nessa rede social digital. O compartilhamento também foi realizado em grupos de WhatsApp de discentes de Programas de Pós-Graduação em Ciência da Informação, em grupos de graduandos em Biblioteconomia, Arquivologia e Administração e em grupos de profissionais atuantes no mercado de trabalho e vinculados a empresas públicas e privadas. Os resultados da pesquisa são apresentados na seção seguinte.

\section{ANÁLISE E INTERPRETAÇÃO DOS DADOS}

As próximas seções têm o propósito de compreender a importância da estratégia omnichannel para a experiência do usuário na rede social digital LinkedIn, respondendo o objetivo deste artigo. Esta seção é organizada com base nas partes que estruturam a ordem das perguntas do questionário aplicado: 'Mapeamento do perfil'; 'Experiência do usuário'; e 'Estratégia omnichannel'. 


\section{Mapeamento do perfil}

Foi realizado o mapeamento do perfil dos/as respondentes, por meio de cinco perguntas relacionadas a: gênero, faixa etária, escolaridade, ocupação e setor de atuação. Nessa parte, buscou-se entender o perfil do público atingido com o nosso questionário via Google Forms, não possuindo relação direta com a estratégia omnichannel ou com a satisfação na experiência do usuário. Pôde-se verificar a predominância do público Mulher Cis em nossa pesquisa, representado por 59,6\% dos respondentes. Em seguida, o público Homem Cis ocupou 39\% das respostas. Também é visto que $0,7 \%$ são do gênero Homem Trans e 0,7\% preferem não declarar o seu gênero. Com relação à faixa etária, a pesquisa abrangeu um público dividido, sendo positivo para o estudo, podendo inferir que a utilização da rede social digital LinkedIn alcança públicos de diferentes gerações. Os dados coletados apresentam que os usuários respondentes estão, em maior parte, entre os 20 a 29 anos, representando 44,1\% e correspondendo à Geração Z ou Geração Google (Cunha, Amaral, \& Dantas, 2015). Em seguida, 25\% estão entre os 30 a 39 anos; 20,6\% entre os 40 a 49 anos; $9,6 \%$ entre os 50 a 59 anos e $0,7 \%$ com 60 anos ou mais.

No que tange à escolaridade, 32,4\% possuem nível de pós-graduação completo; $27,2 \%$ apresentam ensino superior completo; $21,3 \%$ pós-graduação incompleta; $14,7 \%$ ensino superior incompleto; 2,9\% ensino médio completo; 0,7\% mestrado em andamento e 0,7\% ensino médio incompleto. Sendo assim, pôde-se mapear um público especializado no uso da rede social digital LinkedIn. Em relação à ocupação dos respondentes, cerca de 44,1\% estão inseridos no setor privado; $13,2 \%$ são estudantes; $13,2 \%$ estão desempregados, e nesse sentido, o LinkedIn pode contribuir para a recolocação profissional; $11,6 \%$ são servidores públicos; $8,1 \%$ desenvolvem atividades como autônomas; $5,1 \%$ são empresários; $1,5 \%$ trabalham com pesquisas; $0,7 \%$ são aprendizes; $0,7 \%$ são prestadores de serviço público e $0,7 \%$ trabalham e estudam ao mesmo tempo.

Esses dados são relevantes quando se percebe que, na rede social digital LinkedIn, a experiência do usuário pode ser múltipla. Isso porque o LinkedIn pode ser um ambiente digital destinado a públicos que procuram emprego, públicos que procuram estágio, usuários atrás de engajamento para a formação de redes, pesquisadores e pesquisadoras que trabalham na construção de conhecimento científico, profissionais atuantes no setor público e no setor privado, entre outros. Para concluir a primeira parte, relacionada com ao mapeamento do perfil, após o conhecimento da ocupação dos pesquisados, é interessante apresentar o setor com o qual estão vinculados. Pode-se verificar a evidência de três setores: Administrativo, representado por 19,9\% dos respondentes; Educação, com cerca de 19,1\%; e 12,5\% relacionados à Tecnologia da Informação. A seguir, avança-se para a segunda parte do questionário para a satisfação e experiência do usuário com o uso do LinkedIn.

\section{Experiência do Usuário}

Para a experiência do usuário, elaboraram-se sete perguntas sobre a relação do respondente com o uso de computadores e de dispositivos móveis, frequência da utilização da rede social digital LinkedIn, alcance de objetivos adquiridos por meio do LinkedIn, necessidades de acesso, uso e busca de informações por meio das ferramentas dispostas no LinkedIn, entre outras.

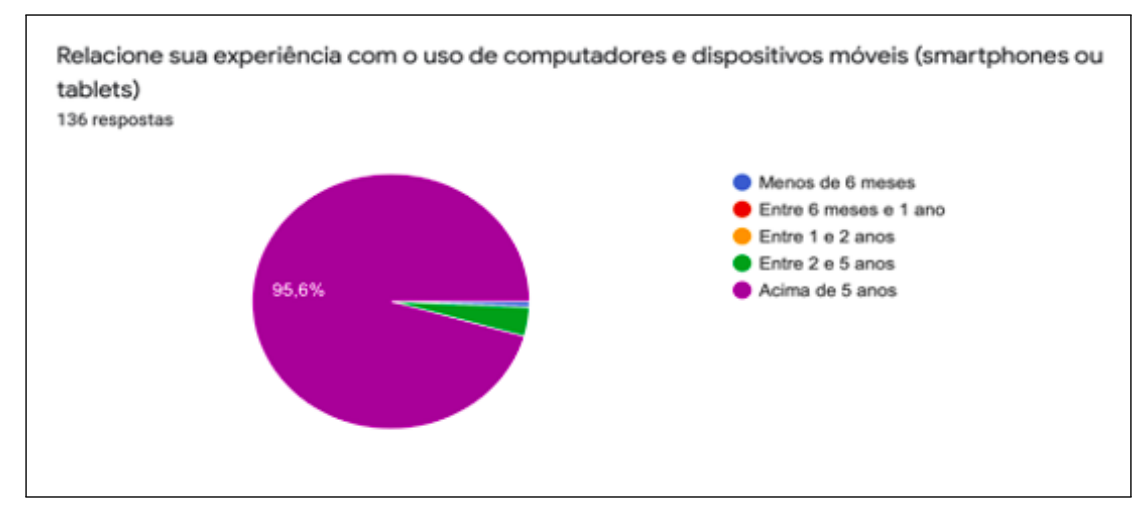

Figura 1. Experiência com o uso de dispositivos tecnológicos.

Fonte: Dados da pesquisa (2021).

Em leitura do Gráfico 1, 95,6\% dos respondentes possuem relação com o uso de computadores e de dispositivos móveis por mais de cinco anos, o que demonstra que os pesquisados têm vivência na utilização de dispositivos tecnológicos, ou seja, são usuários experientes (Cunha et al., 2015). Isso é um resultado interessante, visto que a pesquisa apresenta a experiência omnichannel mediante a satisfação de usuários da rede social digital LinkedIn. Também 3,7\% dos respondentes possuem relação com dispositivos computacionais e móveis no período entre dois e cinco anos; 0,7\% deles possuem essa relação em menos de 6 meses. 


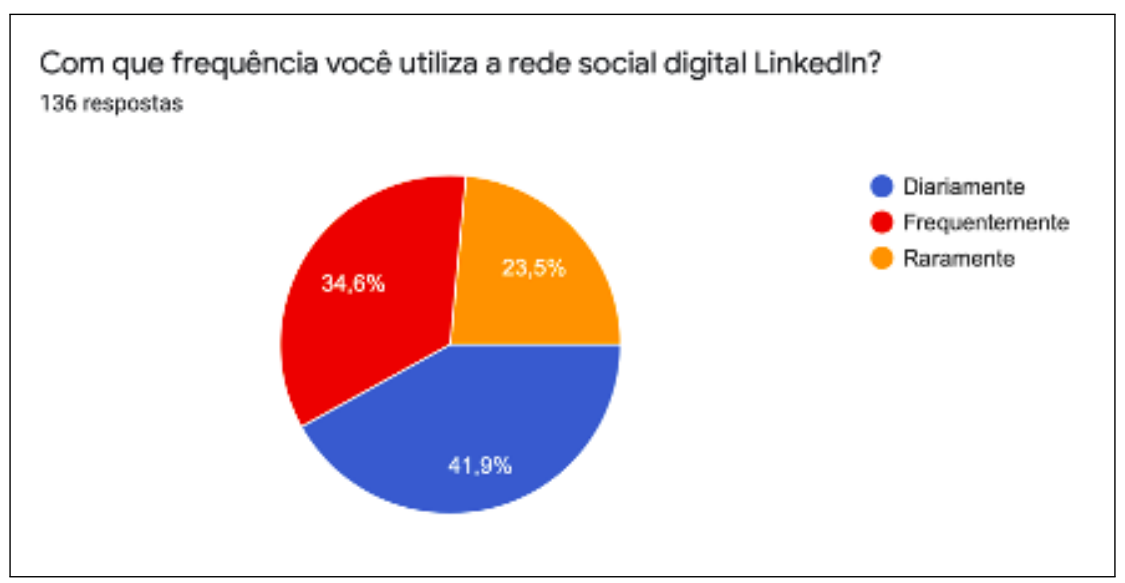

Figura 2. Frequência de utilização da rede social digital Linkedln.

Fonte: Dados da pesquisa (2021).

Constata-se, conforme o Gráfico 2, que 41,9\% dos respondentes utilizam a rede social digital LinkedIn diariamente, $34,6 \%$ utilizam frequentemente e $23,5 \%$ utilizam raramente. Infere-se que esses resultados convergem com a realidade de desemprego que o país passa hodiernamente, haja vista que o LinkedIn é uma rede social que expõe um perfil profissional dos usuários que se cadastram. Muitos indivíduos são encontrados e empregados por empresas através do LinkedIn e seu uso funciona como a inserção de um currículo on-line que pode ser atualizado constantemente (LinkedIn, 2021).

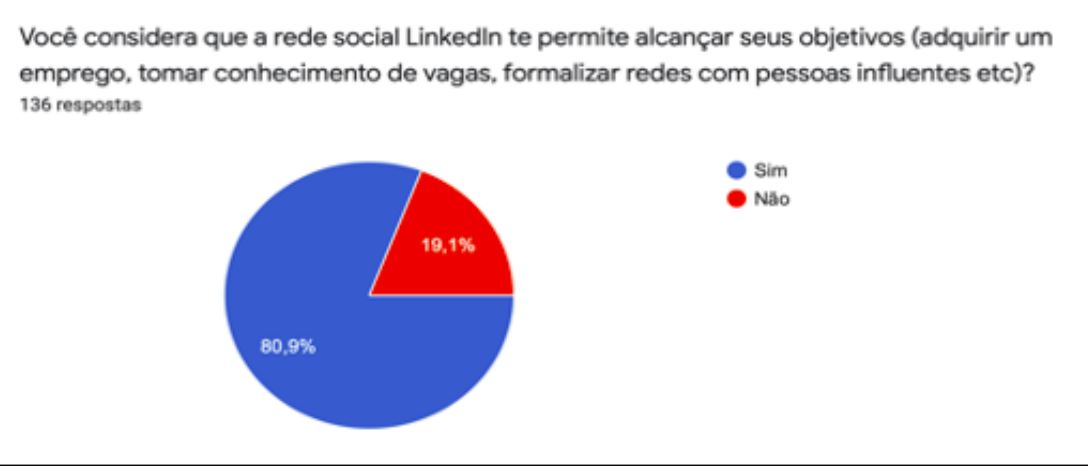

Figura 3. Alcance de objetivos.

Fonte: Dados da pesquisa (2021).

Tem-se então que a maioria do público pesquisado é atuante no uso da rede social digital LinkedIn e com experiência por mais de cinco anos com computadores e dispositivos móveis. Agora, para sinalizar a experiência de satisfação desses usuários, inseriu-se a pergunta disposta no Gráfico 3 sobre o alcance de objetivos pessoais que o LinkedIn pode auxiliar; constatou-se que 80,9\% dos respondentes consideram que o LinkedIn permite adquirir um emprego, conhecer vagas disponíveis no mercado de trabalho, formalizar redes com indivíduos importantes, entre outros. Observou-se também que 19,1\% expõem que esses objetivos não são alcançados, o que representa uma parcela de usuários que não encontra uma boa experiência no alcance de seus objetivos pessoais com a proposta de marketing vendida pelo LinkedIn.

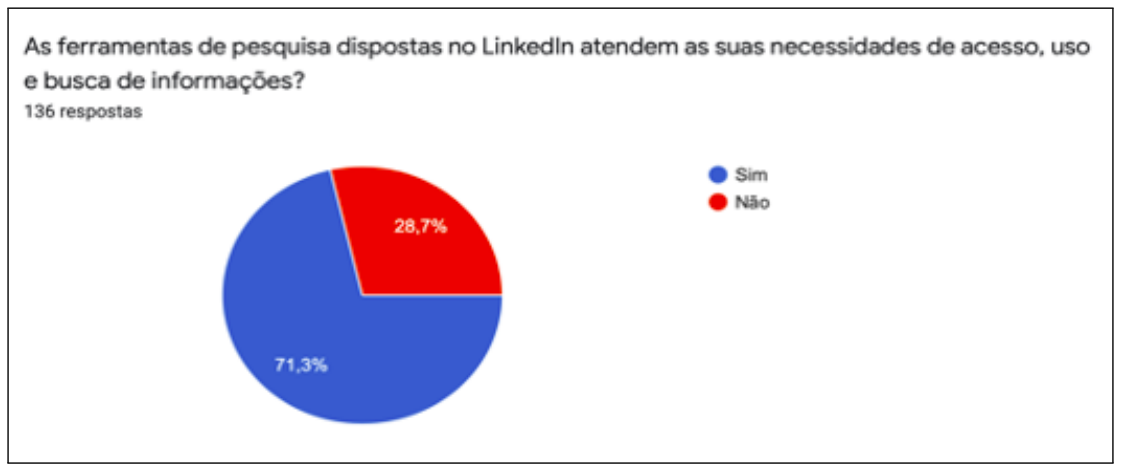

Figura 4. Satisfação quanto às ferramentas de pesquisa do LinkedIn.

Fonte: Dados da pesquisa (2021). 
A parte sobre experiência do usuário procurou conhecer especificamente sobre a satisfação com as ferramentas dispostas na rede social digital LinkedIn. O Gráfico 4 ilustra o questionamento sobre a satisfação proveniente das ferramentas de pesquisa do LinkedIn, quanto às necessidades de acesso, uso e busca de informações no LinkedIn. Constata-se que $71,3 \%$ dos respondentes estão satisfeitos com essas ferramentas e 28,7\% não estão. Infere-se que, mediante os resultados ilustrados no Gráfico 3, a parcela dos 19,1\% que relatam que o LinkedIn não permite o alcance de seus objetivos pode assumir um fio lógico com a parcela de 28,7\% de usuários insatisfeitos com as ferramentas de pesquisa dispostas no LinkedIn, ilustrada no Gráfico 4. Infere-se esse diálogo porque, quando um usuário não alcança seus objetivos num ambiente informacional, esse pode não estar satisfeito com as ferramentas de pesquisa dispostas neste ambiente. Também é provável que essas ferramentas sejam limitadas carecendo de recursos autocomplete ${ }^{3}$, dificultando a recuperação da informação pelo sistema.

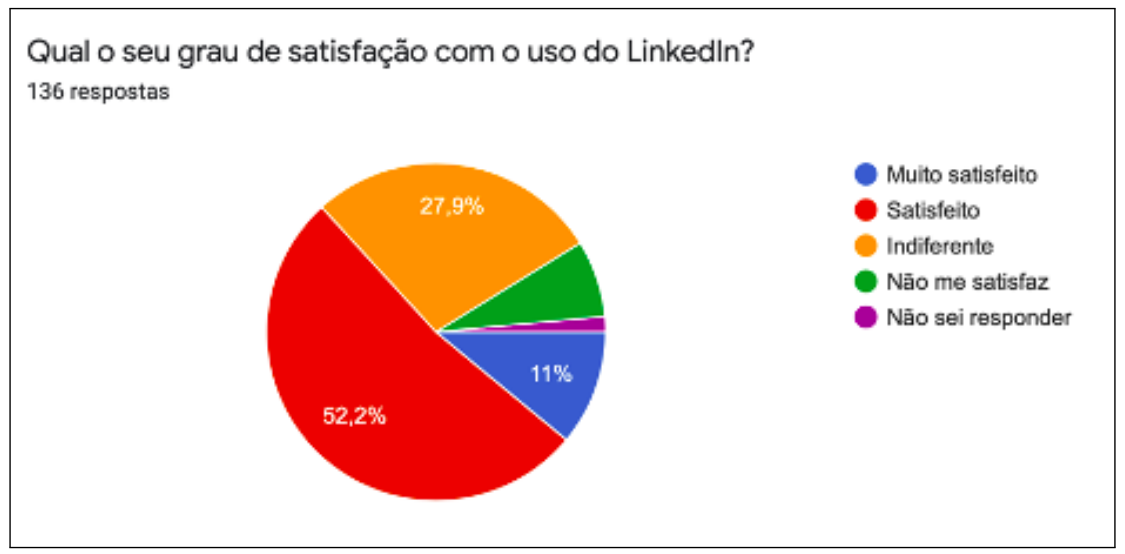

Figura 5. Satisfação quanto ao uso da rede social digital Linkedln.

Fonte: Dados da pesquisa (2021).

Nessa etapa do questionário, avança-se para a satisfação, em geral, sobre o uso da rede social digital LinkedIn. Consonante o Gráfico 5, que ilustra o resultado para o seguinte questionamento "Qual o seu grau de satisfação com o uso do LinkedIn?", constatou-se que $52,2 \%$ dos respondentes estão satisfeitos com o uso da rede social digital LinkedIn, 27,9\% consideram indiferente a satisfação com o uso do LinkedIn, 11\% se consideram muito satisfeitos com o uso do LinkedIn, 7,4\% nos informam que o uso não satisfaz e 1,5\% não sabem responder.

Nota-se que a maioria dos respondentes tem uma boa experiência com o uso do LinkedIn, porém também é necessário um olhar direcionado aos respondentes que responderam "Indiferente", "Não me satisfaz"e "Não sei responder". Certamente, um recurso digital não responde a todas as necessidades de uso dos sujeitos, contudo a maioria do público respondente se satisfaz com o uso do LinkedIn. Dialogando com o Gráfico 3 e com o Gráfico 4, os resultados ilustrados no Gráfico 5 convergem com os 80,9\% dos respondentes que alcançam seus objetivos no uso da rede e com os 71,3\% que estão satisfeitos com a experiência na utilização das ferramentas de pesquisa do LinkedIn.

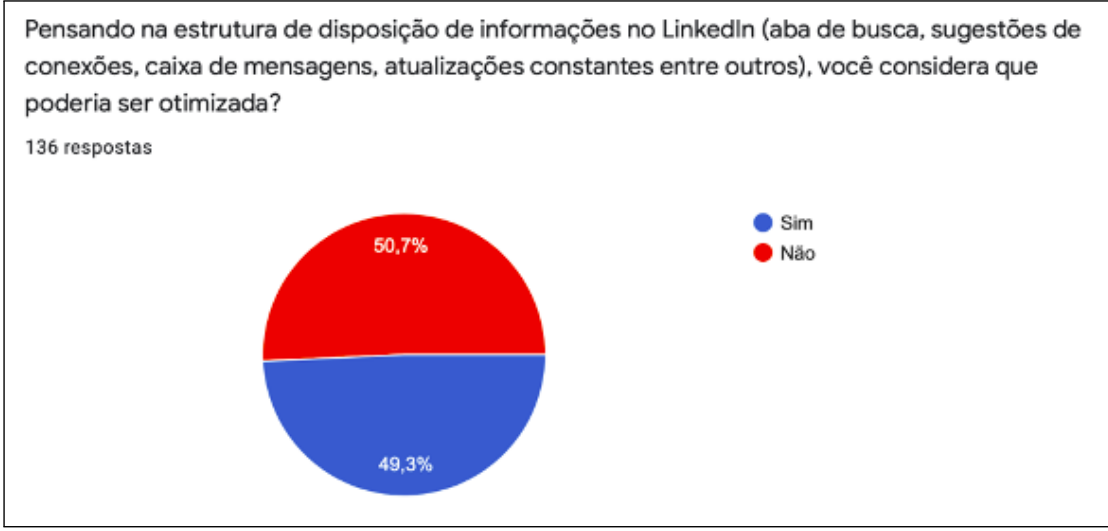

Figura 6. Estruturação de informações no Linkedln.

Fonte: Dados da pesquisa (2021).

Agora, com o Gráfico 6, 50,7\% dos respondentes não consideram que a rede social digital LinkedIn poderia otimizar a sua estrutura de informações. Entende-se como disposição de informações as técnicas e diretrizes

\footnotetext{
${ }^{3} \mathrm{O}$ recurso autocomplete trabalha da seguinte forma: assim que o usuário digita um termo para busca, possíveis previsões de termos ou frases para busca vão aparecendo abaixo da aba de busca (ou caixa de pesquisa). Essas previsões são relacionadas às primeiras letras digitadas, o que auxilia o usuário a direcionar a sua busca ou descobrir recursos ou informações não buscadas inicialmente.
} 
da disciplina de Arquitetura de Informação, isto é, o projeto de ambientes informacionais digitais mediante elementos sistêmicos para o sistema de busca, para o sistema de navegação, para o sistema de rotulagem, para o sistema de organização e para o sistema de representação, como destacam Rosenfeld, Morville, e Arango (2015). Desse modo, para os pesquisados, a Arquitetura de Informação disposta no LinkedIn é adequada e não necessita de otimizações.

Elaborou-se também, para a parte da experiência do usuário, uma pergunta aberta/discursiva, direcionada apenas aos respondentes que marcaram "sim"na questão anterior, ilustrada no Gráfico 6. Obtiveram-se propostas interessantes para possíveis melhorias, como: personalização maior de notificações para o usuário receber; salvar/destacar temas ou postagens interessantes para acompanhamento posterior; informações seletivas, ou seja, o usuário pode selecionar o que é interessante para a sua busca, estabelecendo um filtro do que se deseja encontrar; melhoria na visibilidade das abas e dos links; otimização dos serviços gratuitos; não 'empurrar' os planos pagos ao usuário; filtro para ordem cronológica; informar e dar dicas de cursos na área de atuação do usuário; sugestões de conexões relacionadas com a área de atuação do usuário; customização de ícones para os perfis dos usuários e; melhorar o filtro que é entregue pela aba de busca.

Essas sugestões são bastante relevantes para a otimização da experiência do usuário da rede social digital LinkedIn. Como destacado anteriormente, um ambiente informacional certamente não satisfaz todas as necessidades informacionais dos usuários e, nessa seção, compreendeu-se que existe a possibilidade de melhoria da experiência do usuário no LinkedIn, visto que foram destacados variados recursos no parágrafo anterior mediante o que o usuário deseja e visualiza como necessidade conforme o uso dessa rede social digital. Na seção seguinte, a estratégia omnichannel, terceira parte do nosso questionário, é abordada, dialogando com a experiência do usuário.

\section{Estratégia channel}

Na terceira e última etapa do questionário, buscou-se compreender a percepção dos respondentes quanto à estratégia omnichannel, a fim de se perceber como acontece o relacionamento com o cliente, a partir da integração das informações deste usuário com todos os pontos de interação na relação empresa-consumidor. Para isso, questionaram-se cinco pontos relacionados: à frequência, à adoção, ao compartilhamento, à aceitação e às limitações com o uso dessa estratégia. Apresentam-se esses resultados a seguir.

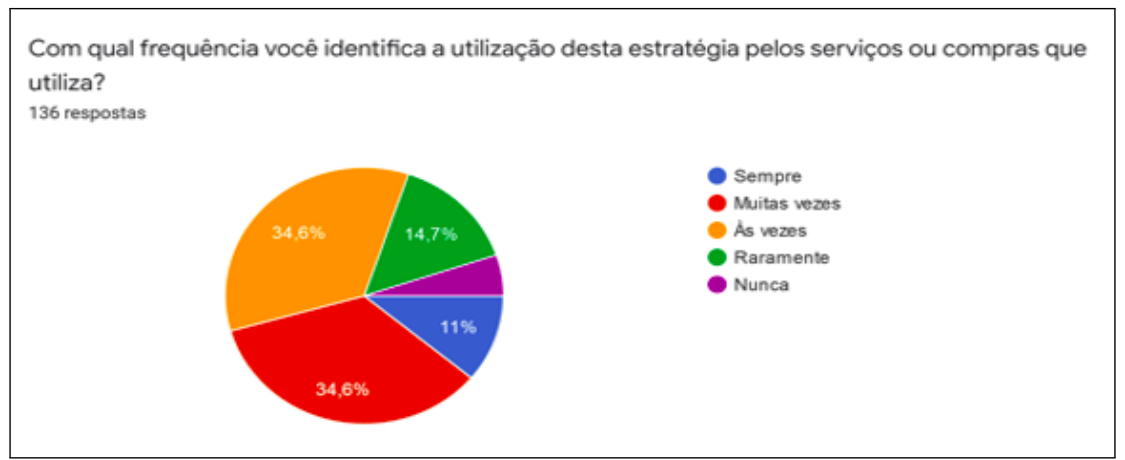

Figura 7. Frequência de utilização da estratégiaomnichannel.

Fonte: Dados da pesquisa (2021).

Com base nos dados evidenciados no Gráfico 7, pôde-se constatar que frequentemente o público está envolvido com a utilização do mecanismo omnichannel em suas buscas informacionais, ou seja, o usuário das informações dispostas no LinkedIn enxerga que a sua experiência na rede está no centro das ações, entre os canais utilizados para a comunicação, sendo o consumidor da informação, também denominado cliente (Corrêa, 2014). Assim, $34,6 \%$ dos respondentes afirmam identificar muitas vezes essa integração entre os canais de comunicação com o consumidor. Já 34,6\% relatam que identificam às vezes; $14,7 \%$ raramente; $11 \%$ sempre e $5,1 \%$ nunca identificam o uso dessa estratégia. 


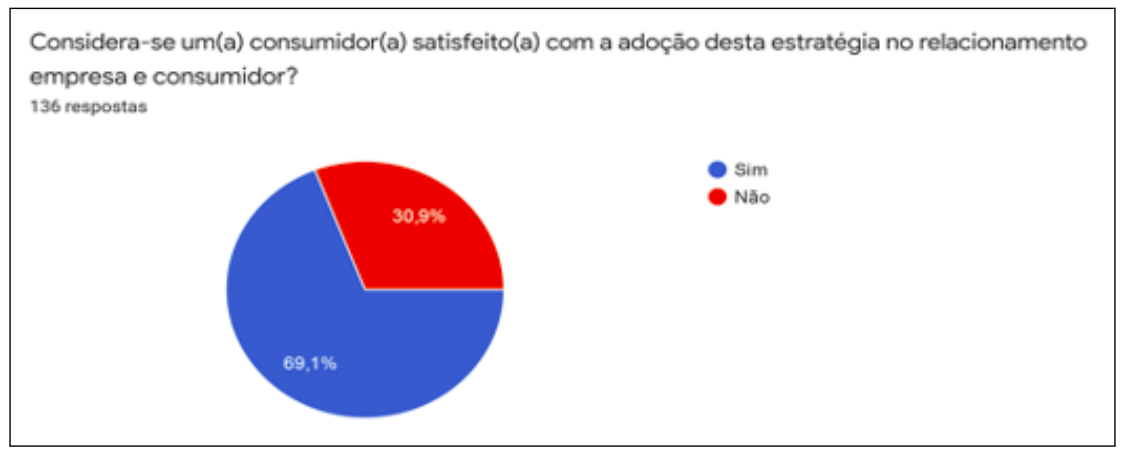

Figura 8. Adoção da estratégia omnichannel. Fonte: Dados da pesquisa (2021).

A fim de compreender o uso da estratégia pelo usuário de informação, o Gráfico 8 demonstra que $69,7 \%$ dos respondentes estão satisfeitos com o uso desta ferramenta, já 30,9\% não consideram a experiência relevante, tal fato poderá ser evidenciado ao questionar o nível de aceitação ou a limitação/barreira digital para utilização. Assim, percebe-se que o consumidor desenvolve um relacionamento com as plataformas que utilizam e que, porventura, disponibilizam este recurso, proporcionando uma experiência particular com nestes canais, fortalecendo o vínculo nos negócios.

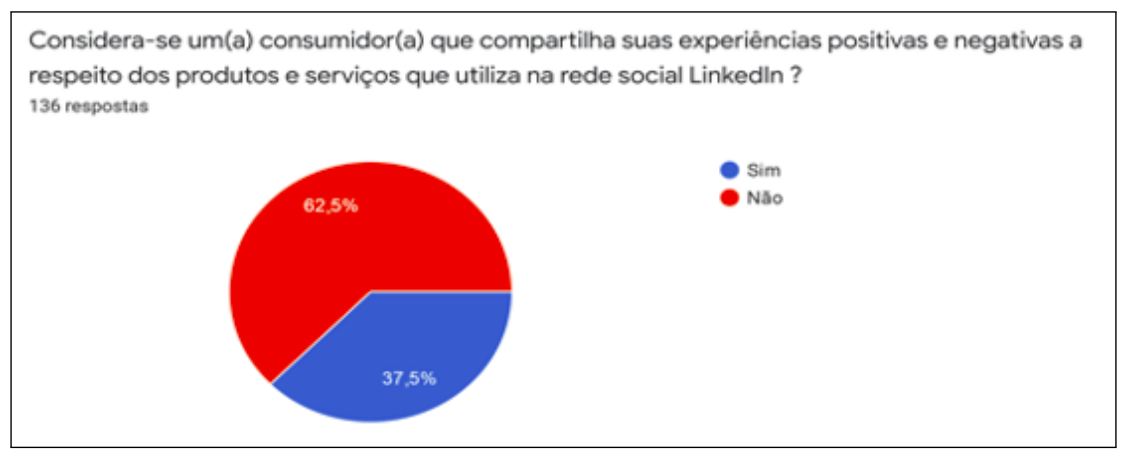

Figura 9. Compartilhamento de experiência com o uso da estratégia omnichannel.

Fonte: Dados da pesquisa (2021).

Com os dados apresentados no Gráfico 9, 62,5\% dos/as respondentes não estão dispostos a compartilharem a experiência de uso com a ferramenta; já 37,5\% mencionam utilizar este recurso. Compreende-se que com a evolução das tecnologias de comunicação, principalmente com a utilização em massa das redes sociais digitais, novas formas de relacionamento surgiram, e uma delas está associada ao compartilhamento com outros usuários da informação. O compartilhamento é uma faceta do comportamento informacional dos usuários na atualidade (Tabosa \& Pinto, 2016). Sendo assim, infere-se que, na pesquisa desenvolvida, o compartilhamento da experiência com a estratégia omnichannel com outros usuários não foi efetivo e, nesse sentido, cabe às organizações desenvolverem ações que estejam alinhadas diretamente com o consumidor, que promovam vantagens competitivas.

O penúltimo questionamento considerou a aceitação dos usuários conforme a experiência com a estratégia omnichannel. É possível analisar os aspectos desejáveis e indesejáveis, em nível emocional e sensorial, que cooperam para a experiência do usuário, conforme os estudos de Rogers, Sharp, e Preece (2013). Nesse sentido, $72,8 \%$ dos respondentes concordam que a estratégia omnichannel está relacionada com os aspectos desejáveis ao afirmarem que o uso é interessante, satisfatório, agradável e motivador. Porém, 27,2\% citam aspectos indesejáveis, ou seja, esses usuários afirmam que o relacionamento é artificial, frustrante, tedioso e sem utilidade.

Por fim, o último questionamento foi sobre a limitação ou barreira digital que acomete a experiência do usuário da informação com a estratégia omnichannel, a fim de compreender os principais gargalos evidentes no processo de relacionamento entre consumidor-empresa. Com base nos estudos de Starec (2006, p. 4), são identificadas barreiras quanto à má comunicação, cultura organizacional, falta de competência e tecnológicas, tais limitações "são móveis e perpassam todas as áreas", e aqui é possível se relacionar a administração, o marketing, o atendimento ao cliente, entre outras. Sendo assim, $43,4 \%$ dos respondentes não possuem limitação/barreira quanto ao uso; é importante destacar que é um fator positivo significa que os usuários envolvidos com a estratégia omnichannel conseguem relacionar e utilizar adequadamente esse mecanismo. Em seguida, 31,6\% sentem dificuldades em relação a má comunicação, ou seja, estes usuários sentem que seus questionamentos não são compreendidos; 14,7\% quanto à cultura organizacional, ou seja, afirmam que as organizações disponibilizam um serviço precário; $8,1 \%$ relacionam a barreiras tecnologias, ou seja, sentem dificuldades na compreensão do funcionamento da estratégia; e 2,2\% afirmam a falta de competência do quadro de pessoal da organização. 


\section{CONSIDERAÇÕES FINAIS}

Como resultado da pesquisa realizada, constatam-se possibilidades de melhorias e compreendem-se as dificuldades enfrentadas e relatadas pelos usuários do LinkedIn respondentes da pesquisa. Em termos de experiência do usuário, a maioria dos participantes da pesquisa demonstra grande satisfação no uso do LinkedIn e, em relação a estratégia omnichannel de gestão integrada, os canais utilizados pelo LinkedIn apresentam esse modelo e a maioria dos usuários percebem essa interligação ao utilizarem a rede social digital. Desse modo, o objetivo traçado foi alcançado. Em resposta à questão de pesquisa, afirma-se que a omnichannel é uma estratégia utilizada pelas organizações para otimizar a experiência do usuário, contribuindo com a usabilidade e podendo conduzi-los para melhores interações com os produtos e serviços buscados, tendo a determinada organização o estabelecimento de relacionamentos com o consumidor aperfeiçoando o fluxo informacional e comunicacional. Para isso, há a interação com chats, ícones, imagens, possibilidades de descoberta de recursos não buscados inicialmente, recursos que façam o usuário se sentir inserido nesse fluxo.

Dentre os maiores problemas enfrentados pelas organizações, está a difícil tarefa de lidar com o fluxo informacional advindo das mais diversas formas de comunicação presentes nos tempos de hoje. Sendo assim, ao lidar com a temática das redes sociais digitais tem-se o estabelecimento de relações entre si, com empresas ou pessoas, de acordo com as suas preferências e particularidades. Na era virtual, as redes sociais digitais são representadas por websites e aplicativos que permitem esse relacionamento e compartilhamento de informações. Frente as discussões evidenciadas na Ciência da Informação, enquanto área proposta a discutir o objeto 'informação' impulsionado pelas Tecnologias de Informação e Comunicação, possibilidades não faltam, tais estudos devem avançar e proporcionar novas temáticas relacionadas aos estudos de usuários, comportamento do consumidor omnichannel, usabilidade e experiência do usuário, segurança da informação em tempos de Lei Geral de Proteção de Dados (LGPD) e temas relacionados à consolidação de uma teoria que possa abordar o percurso de aplicação integrativa da estratégia omnichannel de maneira holística, ou seja, de maneira ampla e não-reducionista. 


\section{REFERÊNCIAS}

Araújo, C. (2018). O que é ciência da informação. Belo Horizonte: KMA.

Borko, H. (1968). Information science: what is it? American Documentation, $19(1), 3-5$.

Buley, L. (2013). The user experience team of one: a research and design survival guide. New York: Rosenfeld Media.

Campos, A. F., \& Oliveira, H. P. C. d. (2020). Traçados teóricos sobre informação e pervasividade. Revista $A C B$ : Biblioteconomia em Santa Catarina, 25(3). Recuperado de https://revista.acbsc.org.br/racb/article/view/1697

Corrêa, E. C. D. (2014). Usuário, não! interagente: proposta de um novo termo para um novo tempo. Encontros Bibli: Revista Eletrônica de Biblioteconomia e Ciência da Informação, 19(41), 23-40. doi: 10.5007/1518-2924.2014v19n41p23

Costa, B., \& Picchi, D. (2017). As relações entre os consumidores do século 21 e as empresas, no âmbito da internet, pelas redes sociais virtuais. Revista FAE, 20(11), 7-26. Recuperado de https://revistafae.fae.edu/revistafae/article/ viewFile/105/433

Cunha, M., Amaral, S., \& Dantas, E. (2015). Manual de estudo de usuários da informação. São Paulo: Atlas.

Ferreira, A. (2018). Contribuições da experiência do usuário para a arquitetura da informação (Tese de doutorado, Universidade Estadual Paulista Júlio de Mesquita Filho, Marília, Brasil). Recuperado de http://hdl.handle.net/11449/ 157487

Fonseca, L. (2017). Estratégia omnichannel: uma nova experiência de consumo. Recuperado de https://rockcontent .com/br/blog/omnichannel/

Guissoni, L. (2017). Omnichannel: uma gestão integrada. Gv Executivo, 16(1), 24-27. doi: 10.12660/gvexec.v16n1.2017.67453

Kotler, P., Kartajaya, H., \& Setiwan, I. (2017). Marketing 4.0: do tradicional ao digital. Rio de Janeiro: Sextante.

Lemos, A. (2010). Cibercultura: tecnologia e vida social na cultura contemporánea (5a. ed.). Porto Alegre: Sulina.

LinkedIn. (2021). Ajuda do linkedin. Recuperado de https://www.linkedin.com/help/linkedin/answers/curr\% C3\%ADculo?hcppcid=homepage

Minayo, M. (2012). Análise qualitativa: teoria, passos e fidedignidade. Ciência \& Saúde Coletiva, 17(3), 621-626. doi: 10.1590/S1413-81232012000300007

Nielsen, J. (1993). Usability engineering. Massachusetts: Morgan Kaufmann.

Rogers, Y., Sharp, H., \& Preece, J. (2013). Design de interação: além da interação humano-computador (3a. ed.). Porto Alegre: Bookman.

Rosenfeld, L., Morville, P., \& Arango, J. (2015). Information architecture: for the web and beyond. s.l.: O'Reilly Media.

Sousa, M. (2012). O acesso a informações e a contribuição da arquitetura da informação, usabilidade e acessibilidade. Informação \& Sociedade: Estudos, 22(esp), 65-76. Recuperado de http://periodicos.ufpb.br/ojs2/index.php/ies/ article/view/13298

Starec, C. (2006). A dinâmica da informação: a gestão estratégica da informação para a tomada de decisão nas organizaçõoes. In C. Starec, E. Gomes \& J. Bezerra. Ges- tão Estratêgica da Informação e Inteligência Competitiva. Saraiva.

Tabosa, H., \& Pinto, V. (2016). Caracterização do comportamento de busca e uso de informação na área de saúde: o modelo de ellis aplicado ao estudo do comportamento informacional de pacientes. Informação \& Sociedade: Estudos, 26(2), 238-255. Recuperado de http://www.ies.ufpb.br/ojs/ index.php/ies/article/view/28583

Tomaél, M., \& Marteleto, R. (2006). Redes sociais: posições dos atores no fluxo da informação. Encontros Bibli: Revista Eletrônica de Biblioteconomia e Ciência da Informação, 11 (esp). Recuperado de https://periodicos.ufsc.br/index .php/eb/article/view/1518-2924.2006v11nesp1p75

Torres, C. (2009). A bíblia do marketing digital. São Paulo: Novatec.

Van Dijck, J. (2016). La cultura de la conectividade: una historia critica de las redes sociales. Tres Cantos: Siglo Veintiuno Editores.

Verhoef, P., Kannan, P., \& Inman, J. (2015). From multi-channel retailing to omni channel retailing: introduction to the special issue on multi-channel retailing. Journal Of Retailing, 91(2), 174-181. Recuperado de https://courses.helsinki.fi/sites/default/files/course -material/4482601/17.3_JR\%20Special\%20issue.pdf
Como citar este artigo (APA):

Ramos, B. S., Campos, A. F., Paiva, E. B., Freire, G. H. de, \& Sousa, M. R. F. de (2021). Estratégia omnichannel e a experiência do usuário: um estudo na rede social digital LinkedIn. AtoZ: novas práticas em informação e conhecimento, 10(3), 1 - 11. Recuperado de: http://dx.doi.org/10.5380/atoz.v10i3.81054 